\title{
Confirmation via Analogue Simulation: What Dumb Holes Could Tell us About Gravity
}

\author{
Radin Dardashti ${ }^{* 1}$, Karim Thébault ${ }^{\dagger 1}$, and Eric Winsberg ${ }^{\ddagger 2}$ \\ ${ }^{1}$ Munich Center for Mathematical Philosophy, Ludwig Maximilians Universität Munich \\ ${ }^{2}$ Philosophy Department, University of South Florida
}

August 14, 2014

\begin{abstract}
In this paper we argue for the existence of analogue simulation as a novel form of scientific inference with the potential to be confirmatory. This notion is distinct from the modes of analogical reasoning detailed in the literature, and draws inspiration from fluid dynamical 'dumb hole' analogues to gravitational black holes. For that case, which is considered in detail, we defend the claim that the phenomena of gravitational Hawking radiation could be confirmed in the case that its counterpart is detected within experiments conducted on diverse realisations of the analogue model. A prospectus is given for further potential cases of analogue simulation in contemporary science.
\end{abstract}

\section{Contents}

1 Introduction $\quad 2$

2 Physical Background 3

2.1 Hawking Radiation in Semiclassical Gravity . . . . . . . . . . . . . . . 3

2.2 Modelling Sound in Fluids . . . . . . . . . . . . . . . . 5

2.3 The Acoustic Analogue Model of Hawking Radiation . . . . . . . . . . . 7

3 Simulation and Analogy in Physical Theory 9

3.1 Analogical Reasoning and Analogue Simulation . . . . . . . . . . . . . . 9

3.2 Confirmation via Analogue Simulation . . . . . . . . . . . . . . . . . . . . . 12

3.3 Recapitulation . . . . . . . . . . . . . . . . . 16

4 The Sound of Silence: Analogical Insights into Gravity 18

4.1 Experimental Realisation of Analogue Models . . . . . . . . . . . . . . . . . 18

4.2 Universality and the Hawking Effect . . . . . . . . . . . . . . . . . . 20

4.3 Confirmation of Gravitational Hawking Radiation . . . . . . . . . . . . 23

5 Prospectus $\quad 25$

*email: Radin.Dardashti@Irz.uni-muenchen.de

${ }^{\dagger}$ email: karim.thebault@gmail.com

‡email: winsberg@usf.edu 


\section{Introduction}

Philosophical analysis of science is always, at least partially, held hostage to the fortunes of scientific practice. As the ways in which scientists do science evolve, so must the models of science put forward by philosophers, else the discipline will inevitably decline into irrelevance. Yet, mere empty imitation would be no more constructive a pursuit. To remain relevant the philosophy of science must retain its normative role regarding scientific practice, even as this practice evolves. Here we will articulate a refinement and extension of existent analysis of the role of analogies in science (Keynes 1921, Hesse 1964, Hesse 1966, Bartha 2010, Bartha 2013) inspired by fluid mechanical 'dumb hole' analogues to gravitational black holes (Unruh 1981, Novello, Visser, and Volovik 2002, Barceló, Liberati, Visser, et al. 2005, Unruh 2008). Our central claim, which we take to be both bold and well founded, is that this case exemplifies a notion of analogue simulation that, unlike other species of analogical reasoning, has the potential to provide a conduit for confirmation. Trading on a robust syntactic isomorphism between the relevant modelling frameworks, analogue simulation allows certain inaccessible phenomenology in the target system to be probed by experimentation on the analogue. Given further model-external and empirically grounded arguments, this then allows us to 'confirm' the existence of novel phenomenology in the target system via the observation of its correlate in the analogue. The potential importance of this claim is particularly startling in the context of our chosen example since Hawking radiation is among the gravitational phenomena that 'dumb holes' have the capacity to simulate, and by our lights confirm. Thus, if our analysis is correct, the quantum phenomenology of black holes is potentially within reach of contemporary experimental research in analogue gravity (Carusotto, Fagnocchi, Recati, Balbinot, and Fabbri 2008).

Our arguments regarding confirmation via analogue simulation do, however, cut both ways. Given the requirement for additional model-external and empirically grounded arguments, on its own the analogue simulation of some phenomena is not taken to be confirmatory. Thus, claims made in the literature (Weinfurtner, Tedford, Penrice, Unruh, and Lawrenc 2013) regarding the 'verification' of classical aspects of Hawking radiation must, for the moment, be treated carefully. On our analysis, confirmation of Hawking radiation via analogue simulation can only be established given the acceptance of a chain of reasoning involving universality arguments in combination with diverse realisations of the counterpart effect. These stringent conditions provide both the normative thrust of our analysis of the case in hand and a framework for the investigation of further cases. In general terms, our aim in what follows is to establish the existence of a distinct notion of analogue simulation, and then to provide conditions for this mode of scientific inference to be confirmatory. Whether or not there exist cases in which these conditions in fact obtain remains to be seen: further progress in empirical science is needed before one could justifiably claim confirmation of Hawking radiation via analogue simulation.

In the following Section 2 we will briefly review the physical background necessary for a basic understanding of: Hawking radiation in semi-classical gravity ( $\$ 2.1)$; the modelling of sound in fluids (\$2.2); and the acoustic analogue model of Hawking radiation (§2.3). Section 3 then contains explication of analogue simulation and our claim that it can provide a means for confirmation. In the first subsection (§3.1) we review the traditional notion of analogical reasoning, introduce a framework for understanding analogue simulation, and then contrast the two. In the following subsection (\$3.2) we deal with the problem of justifying the inferences necessary for analogue simula- 
tion to enable confirmation. Our key idea is that in certain circumstances predictions concerning inaccessible phenomena can be confirmed via an analogue simulation in a different system. As we shall see, one is only justified in making such claims once one has established additional empirically grounded and model-external arguments for the accuracy and robustness of the relevant modelling frameworks and syntactic isomorphism within the domains involved. We conclude Section 3 with a recapitulation of the key distinctions and their relevance to issues of confirmation (§3.3). The problems of experimental realisation of Hawking radiation ( $\$ 4.1)$ and of finding the relevant model-external, empirically grounded arguments for the dumb hole/black hole case (§4.2) then become our main occupation. In completion of our argument, we present the case for the dumb hole/black hole correspondence offering us the possibility for confirmation of Hawking radiation via analogue simulation (§4.3). We conclude by offering a prospectus for extension of the idea of analogue simulation to other areas of science, and give a short sketch of one case of particularly obvious relevance $(\S 5)$.

\section{Physical Background}

\subsection{Hawking Radiation in Semiclassical Gravity}

In this section we will give a brief overview of the basis behind Hawking's famous calculation demonstrating that black holes are associated with a particle flux connected with a characteristic 'black hole temperature' (Hawking 1975). Before we do this we should note that a number of rich and important interpretational issues regarding such Hawking radiation are yet to be fully resolved. Most troublingly, the sense in which the radiation is localisable to the interior of the black hole, the event horizon, or the exterior of the black hole, is far from clear. Hawking's calculation does not provide a causal mechanism for the radiation and thus, to a degree, renders the phenomena rather mysterious. This notwithstanding, the original thermal model for Hawking radiation has proved 'remarkably robust' under the inclusion of various complicating factors (Leonhardt and Philbin 2008, Thompson and Ford 2008), and thus can be taken as sufficient for our purposes.

Our starting point is a semi-classical approach to gravity where we consider a quantum field within a fixed spacetime background. We will follow the standard treatment ${ }^{1}$ and consider the simplest possible model with a scalar field $\phi$ considered in 1+1 Minkowskian background. The classical wave equation for a free scalar field is simply given by $g^{a b} \nabla_{a} \nabla_{b} \phi=0$ where the scalar field is a quantum operator, i.e., obeys the canonical equal-time commutation relations and acts on a suitably constructed Hilbert space. We then expand the scalar field in a basis of orthonormal plane wave solutions:

$$
\phi=\int d \omega\left(a_{\omega} f_{\omega}+a_{\omega}^{\dagger} f_{\omega}^{*}\right)
$$

where $f_{\omega}=\frac{1}{\sqrt{2}} e^{-i(\omega t-k x)}$ and $a_{\omega}, a_{\omega}^{\dagger}$ are operators now satisfying $\left[a_{\omega^{\prime}}, a_{\omega}^{\dagger}\right]=\delta\left(\omega^{\prime}-\omega\right)$, for some frequency $\omega$ and real constant $k$. The operators $a_{\omega}^{\dagger}$ and $a_{\omega}$ can thus be interpreted as creation and annihilation operators. We can now consider the vacuum state for the scalar field at past null infinity, $\mathcal{J}^{-}$, to be the 'in' state, and define it in

\footnotetext{
${ }^{1}$ See (Mukhanov and Winitzki 2007) for an elementary introduction.
} 
the usual way as $a_{\omega}|0\rangle_{\text {in }}=0$ for all $\omega>0$. It is natural to then also define the number operator for the 'in' state at each frequency $\omega$ as $N_{\omega}^{i n}=a_{\omega}^{\dagger} a_{\omega}$.

Now, consider an alternative set of solutions $\left\{p_{\omega}, p_{\omega}^{*}\right\}$ which form a complete orthonormal basis and with respect to which we can also expand the scalar field:

$$
\phi=\int d \omega\left(b_{\omega} p_{\omega}+b_{\omega}^{\dagger} p_{\omega}^{*}\right)
$$

with $\left[b_{\omega^{\prime}}, b_{\omega}^{\dagger}\right]=\delta\left(\omega^{\prime}-\omega\right)$. We use these creation and annihilation operators defined in this new basis to specify the properties of the 'out' state at future null infinity, $\mathcal{J}^{+}$, via $b_{\omega}|0\rangle_{\text {out }}=0$, and $N_{\omega}^{\text {out }}=b_{\omega}^{+} b_{\omega}$. Since the massless scalar field is completely determined by its Cauchy data on either of the surfaces $\mathcal{J}^{-}$or $\mathcal{J}^{+}$, it can be expressed in the form (1) or (2) everywhere. This means that we can transform the $f_{\omega}$ solutions in terms of the $p_{\omega}$ solutions, and vice versa, via the Bogoliubov transformations:

$$
f_{\omega}=\int d \omega^{\prime}\left(\alpha_{\omega \omega^{\prime}}^{*} p_{\omega^{\prime}}-\beta_{\omega \omega^{\prime}} p_{\omega^{\prime}}^{*}\right) \quad p_{\omega}=\int d \omega^{\prime}\left(\alpha_{\omega \omega^{\prime}} f_{\omega^{\prime}}+\beta_{\omega \omega^{\prime}} f_{\omega^{\prime}}^{*}\right)
$$

where $\alpha_{\omega \omega^{\prime}}$ and $\beta_{\omega \omega^{\prime}}$ are the Bogoliubov coefficients given by the inner products $\alpha_{\omega \omega^{\prime}}=\left(p_{\omega}, f_{\omega^{\prime}}\right)$ and $\beta_{\omega \omega^{\prime}}=-\left(p_{\omega}, f_{\omega^{\prime}}^{*}\right)$. Similarly, the Bogoliubov coefficients relate the relevant mode operators to each other, e.g.

$$
b_{\omega}=\int d \omega^{\prime}\left(\alpha_{\omega \omega^{\prime}}^{*} a_{\omega^{\prime}}-\beta_{\omega \omega^{\prime}}^{*} a_{\omega^{\prime}}^{\dagger}\right) .
$$

These results give us the basis to calculate the expectation value of the out number operator for the in vacuum state:

$$
{ }_{i n}\left\langle 0\left|\left(N_{\omega}^{o u t}\right)\right| 0\right\rangle_{\text {in }}={ }_{i n}\left\langle 0\left|b_{\omega}^{\dagger} b_{\omega}\right| 0\right\rangle_{\text {in }}=\int d \omega^{\prime}\left|\beta_{\omega \omega^{\prime}}\right|^{2}
$$

Thus, even in this simple model for semi-classical gravity we can see that the initial vacuum state of a scalar field in a classical spacetime need not appear as a vacuum state to observers at positive null infinity: it may contain a flux of 'out-particles' which one can calculate simply by determining the relevant coefficient $\beta_{\omega \omega^{\prime}}$. In the case where the two solutions are related to each other by a Lorentz transformation, both observers associated with the solutions will of course agree on the number of particles observed and (5) will vanish.

What Hawking's calculation (Hawking 1975, pp. 204-213) shows is that, for a spacetime which features the establishment of an event horizon via gravitational collapse leading to a black hole, one can derive the asymptotic form of the relevant coefficients $\beta_{\omega \omega^{\prime}}$, and show that it depends only upon the surface gravity of the black hole and in the long-time limit not on the details of the gravitational collapse. ${ }^{2}$ We can define surface gravity, $\kappa$, in terms of the magnitude of the acceleration, with respect to Killing time, of a stationary zero angular momentum particle just outside the horizon (Jacobson 1996). This is, more intuitively put, the force per unit mass that must be applied at infinity in order to hold the particle on its path. For a nonrotating neutral black hole the surface gravity is given by $\frac{1}{4 M}$, where $M$ is the black hole mass.

\footnotetext{
${ }^{2}$ In essence the calculation follows the same lines as that for the scalar field $\phi$ in $1+1$ Minkowskian space, only the fields are also expanded for the surface defined by the event horizon. Importantly one does not need to consider a quantum field in a curved spacetime since the field operators are only evaluated in the asymptotic regime which is presumed to be flat.
} 
The role of surface gravity in black hole thermodynamics is almost identical to that of temperature in conventional thermodynamics. In fact, we define the black hole temperature, $T_{B H}$, in terms of the surface gravity. Hawking's calculation thus gives a general demonstration that there is a connection between the intrinsic thermodynamic properties of a (non-eternal) black hole (or at least its horizon) and a non-zero particle flux at late times. The precise relation takes the form:

$$
\left\langle N_{\omega}^{\text {Black Hole }}\right\rangle=\frac{1}{e^{\frac{2 \pi \omega}{\hbar \kappa}}-1} \quad T_{B H}=\hbar \kappa / 2 \pi
$$

where we now simply refer to the evaluation of the expectation value of the late time particle number operator relative to the initial vacuum as the 'black hole' particle flux. We thus see that the basis behind Hawking's derivation of Hawking radiation is a very general one: it requires us only to consider simple features of quantum vacuum states when evaluated in classical spacetime backgrounds which feature Killing horizons. It is a kinematical effect not a dynamical one. The Einstein equation is not used anywhere in the calculation. As noted above, such a derivation of what is, after all, a highly non-trivial physical effect seems a little unsatisfactory from a causal view point and it has some severe problems some of which we discuss later ${ }^{3}$. However, for our purpose of investigating analogue models of Hawking radiation it will prove best to think about the gravitational derivation in precisely such general and non-microcausal terms.

\subsection{Modelling Sound in Fluids}

Sound is generally understood as a small vibratory or wavelike disturbance in a medium. Classical physics deals with such acoustic phenomena in both fluids and solids and, within certain realms of application, gives an empirically adequate description. A simple but powerful classical acoustic model (Landau and Lifshitz 1987, $\S 64)$ is that where the fluid is taken as a continuous, compressible, inviscid medium and sound is understood as a longitudinal oscillatory motion with small amplitude within the medium. Since the fluid in such a model is treated as a continuous medium, 'points' within it are really volume elements that are presumed to contain a very large number of fluid molecules. The volume elements are taken to be very small with respect to the overall fluid volume, and very large with respect to the inter-molecular distances, meaning that the model is only valid in a particular window of applicablity determined by the size of the fluid and relevant molecular distances. The first fundamental equation within this model of a fluid is the continuity equation. This is simply an expression of the conservation of matter, and takes the form:

$$
\frac{\partial \rho}{\partial t}+\nabla \cdot(\rho \vec{v})=0
$$

where $\rho$ is the mass density of the fluid at a particular point, and $\vec{v}$ is the velocity of the fluid volume element. A third quantity, in addition to density and velocity, that we use to characterise the fluid is the pressure, $p$. The total force due to the surrounding fluid acting on a unit of fluid - i.e. a fluid 'particle' - is given by $-\nabla p$ and this, by Newton's second law, must be equal to the rate of change in velocity of the fluid

\footnotetext{
${ }^{3}$ For a recent discussion of some of these problems see (Unruh 2014).
} 
particle relative to space. These considerations lead us to the second fundamental equation, the Euler equation:

$$
\rho\left(\frac{\partial \vec{v}}{\partial t}+(\vec{v} \cdot \nabla) \vec{v}\right)=-\nabla p
$$

The simultaneous solution of these two equations gives us a basic model for the entire fluid flow, which is characterised in a given situation by the triple of functions $\rho(x, y, z, t), p(x, y, z, t)$ and $\vec{v}(x, y, z, t)$.

We understand a sound wave in terms of an alternate compression and rarefaction at each point in the fluid, and can produce a model for sound traveling through a fluid in terms of the movement of small fluctuations around the equilibrium density and pressure. Explicitly we can consider linearized fluctuations around the exact solutions of the form:

$$
\begin{aligned}
\rho(t, x, y, z) & =\rho_{0}(t, x, y, z)+\epsilon \rho_{1}(t, x, y, z)+\ldots \\
p(t, x, y, z) & =p_{0}(t, x, y, z)+\epsilon p_{1}(t, x, y, z)+\ldots \\
\vec{v}(t, x, y, z) & =\vec{v}_{0}(t, x, y, z)+\epsilon \vec{v}_{1}(t, x, y, z)+\ldots
\end{aligned}
$$

The equations of motion of the fluctuations described by $\left(\rho_{1}, p_{1}, \vec{v}_{1}\right)$ is then precisely the model for sound propagation in fluids. Such a model (given additional simplifying assumptions) allows us to reproduce a number of important fluid acoustic phenomena such as resonance and reflection/refraction of sound waves in mediums composed of homogenous layers of different fluids (Landau and Lifshitz 1987, §65-81) .

As emphasised already, the model's applicability depends crucially upon the fluid volume, volume unit size, and inter-molecular distances all being orders of magnitude apart. Further limits on the applicability are also given by the speed at which both the sound wave and the fluid itself are travelling: if either of these are comparable to the speed of light then a relativistic fluid dynamical model would be needed (Landau and Lifshitz 1987, §134). Now, the point crucial to our analysis is that even if we assume that in these respects the model is within its domain of applicability, there is still scope for it to need modification due to quantum effects. If we consider a Bose-Einstein condensate (realised, for example in terms of a superfluid such as liquid $\mathrm{He}^{4}$ close to absolute zero) then certain assumptions of our simple model for sound propagation in fluids necessarily breakdown, but certain do not. In particular, within certain regimes, the quantum field corresponding to the Bose-Einstein condensate can be separated into a bulk flow component and linearized fluctuations. We can then still treat the bulk as an essentially classical, macroscopic fluid similar to those discussed above, ${ }^{4}$ but we now treat the linearized fluctuations (i.e. sound) as fundamentally quantum mechanical. That is, under certain conditions, for certain very low temperature fluids, even if the bulk fluid flow is still treated classically, it becomes appropriate to treat the elementary excitations as quanta of the sound wave - i.e. as phonons akin to those used in the quantum mechanical descriptions of sound in crystalline solids. This is a strikingly similar type of semi-classical approximation to that made by Hawking in the context of black holes as discussed above. In the next section we will see that the similarity between acoustic and gravitational models can, in certain circumstances, be

\footnotetext{
${ }^{4}$ In fact the bulk fluid equations can be shown to reduce to a continuity equation plus an Euler equation which are completely equivalent to those of a classical inviscid fluid apart from the existence of the quantum potential term in the latter (Barceló, Liberati, Visser, et al. 2005, p.60).
} 
arranged so that they have exactly the same mathematical structure. This will lead the way to the introduction of the notion of Analogue Simulation in Section 3.

\subsection{The Acoustic Analogue Model of Hawking Radiation}

Following (Unruh 1981) and (Barceló, Liberati, Visser, et al. 2005), let us consider a simple model for a classical fluid along the lines discussed above (i.e., a continuous, compressible, inviscid medium in a non-relativistic regime), but with the additional assumptions that the fluid is barotropic and locally irrotational. Barotropic simply means the pressure is a function of the density (or vice versa), and so equates to imposing the condition $p=p(\rho)$. Locally irrotational means that their are no vortices in the fluid and equates to imposing the condition $\nabla \times \vec{v}=0$, which implies $\vec{v}=\nabla \psi$, where we have introduced the velocity potential $\psi$. The barotropic and irrotational conditions allow us to much simplify Euler's equation so that it reduces to a form of the Bernoulli equation. We can then consider the linearisation of the solutions to this equation of motion for the entire fluid about a background, $\left(\rho_{0}, p_{0}, \psi_{0}\right)$,

$$
\begin{aligned}
\rho(t, x) & =\rho_{0}(t, x)+\epsilon \rho_{1}(t, x)+\ldots \\
p(t, x) & =p_{0}(t, x)+\epsilon p_{1}(t, x)+\ldots \\
\psi(t, x) & =\psi_{0}(t, x)+\epsilon \psi_{1}(t, x)+\ldots
\end{aligned}
$$

Again we identify the sound waves in the fluids with the fluctuations $\left(\rho_{1}, p_{1}, \psi_{1}\right)$ about the background, which is interpreted as bulk fluid motion. The linearised version of the continuity equation (together with the barotropic condition) then allows us to write the equation of motion for the fluctuations as :

$$
\frac{\partial}{\partial t}\left(\frac{\rho_{0}}{c_{\text {sound }}^{2}}\left(\frac{\partial \psi_{1}}{\partial t}+\overrightarrow{v_{0}} \cdot \nabla \psi_{1}\right)\right)=\nabla \cdot\left(\rho_{0} \nabla \psi_{1}-\frac{\rho_{0} \overrightarrow{v_{0}}}{c_{\text {sound }}^{2}}\left(\frac{\partial \psi_{1}}{\partial t}+\overrightarrow{v_{0}} \cdot \nabla \psi_{1}\right)\right)
$$

This equation can be rewritten as:

$$
\frac{1}{\sqrt{-g}} \frac{\partial}{\partial x_{\mu}}\left(\sqrt{-g} g^{\mu \nu} \frac{\partial}{\partial x^{v}} \psi_{1}\right)=0
$$

where we have defined the acoustic metric

$$
g_{\mu \nu}^{\text {acoustic }}=\frac{\rho_{0}}{c_{\text {sound }}}\left(\begin{array}{ccc}
-\left(c_{\text {sound }}^{2}-v_{0}^{2}\right) & \vdots & -\left(v_{0}\right)_{j} \\
\cdots & \cdot & \cdots \\
-\left(v_{0}\right)^{i} & \vdots & \delta_{i j}
\end{array}\right)
$$

Note that (10) reduces to the simple free scalar field equation we considered in Section 2.1 for a $1+1$-Minkowski metric. We thus see that the propagation of sound in a fluid can be understood as being governed by an acoustic metric of the form $g_{\mu v}$. The acoustic perturbations couple only to the effective acoustic metric and not to the physical spacetime metric which describes the spacetime in which the fluid exists. Formally the acoustic metric describes a (3+1)-dimensional Lorentzian (pseudo-Riemannian) geometry and it depends algebraically on the density, velocity of flow, and local speed of sound in the fluid meaning it is constrained to have at most three degrees of freedom per point in spacetime. 
The close similarity between the acoustic case and gravity can be seen immediately if we consider the Schwarzschild metric in Painleve-Gullstrand coordinates (within which the Schwarzschild geometry is written such a way that space is flat, even though spacetime is curved). This takes the form:

$$
g_{\mu \nu}^{\text {Schwarzschild }}=\left(\begin{array}{ccc}
-\left(c_{0}^{2}-\frac{2 G M}{r}\right) & \vdots & -\sqrt{\frac{2 G M}{r}} \vec{r}_{j} \\
\ldots & \cdot & \cdots \\
-\sqrt{\frac{2 G M}{r}} \vec{r}_{i} & \vdots & \delta_{i j}
\end{array}\right)
$$

This similarity can be transformed into an isomorphism (up to a factor) under certain very specific conditions. Explicitly, following (Novello, Visser, and Volovik 2002), we make the restrictions that: i) $c_{\text {sound }}$ is a position-independent constant; ii) the fluid moves radially with a velocity profile $v_{\text {fluid }}=\frac{1}{\sqrt{r}}$; and iii) the background density $\rho_{0}$ is a position-independent constant. Given these requirements our fluid metric becomes such that it is proportional to the Schwarzschild metric above. The role of the black hole event horizon is now played by the effective acoustic horizon where the inward flowing magnitude of the radial velocity of the fluid exceeds the speed of sound. The black hole is replaced by a dumb hole.

Thus we have a methodology for simulating (in a sense to be discussed more fully shortly) a classical black hole using fluid mechanics. This is in of itself a rather impressive result. However, one is able to stretch the fluid/gravity analogy even further and consider both classical and quantum mechanical acoustic phenomena within the fluid as analogies to radiative phenomena within a black hole spacetime. The relevant calculation for the acoustic case proceeds in precisely the same manner as the Hawking calculation considered above only with the scalar field corresponding to sound, and in this case leads to a relation between the late time sound flux associated with the dumb hole, and the 'surface gravity' of the acoustic horizon. The latter is simply equal to the physical acceleration of the fluid as it crosses the event horizon, $a_{\mathrm{fluid}}$, and is given by an expression of the form:

$$
\kappa=c_{\text {sound }}\left|\frac{\partial v}{\partial n}\right|=a_{\text {fluid }}
$$

Where $\frac{\partial}{\partial n}$ is the normal derivative of the fluid velocity as it crosses the event horizon. Given this, we can make numerical estimates for the acoustic Hawking Temperature, $T_{H}^{\text {acoustic }}$, of a dumb hole for any given fluid. And since, unlike for black holes, we have experimental access to fluids, it means that there is in principle a means for testing the predicted Hawking radiation of a dumb hole.

We will return to the various interesting issues surrounding experimental observation of Hawking radiation via analogue models in Section 4 . There we will consider the extant experiments to test for fluid mechanical Hawking radiation, and also give some details regarding possible future experiments using different analogical models. We will also consider the implications of the short length scales breakdown of the analogue models for our understanding of the possible Planckian breakdown of the gravitation model. This will finally lead into a discussion of Hawking radiation in the context of universality, and allow us to understand precisely what dumb holes could tell us about gravity. Before then, in the following section, we will consider the implications of the analogue models we have been discussing for the general analysis of simulation and analogy in physical theory. 


\section{Simulation and Analogy in Physical Theory}

\subsection{Analogical Reasoning and Analogue Simulation}

Arguments by analogy are very common in both science and philosophy. An oftcited example is an argument offered by the Scottish Philosopher Thomas Reid for the existence of life on other planets:

Thus, we may observe a very great similitude between this earth which we inhabit, and the other planets, Saturn, Jupiter, Mars, Venus, and Mercury. They all revolve round the sun, as the earth does, although at different distances, and in different periods. They borrow all their light from the sun, as the earth does. Several of them are known to revolve round their axes like the earth, and, by that means, must have a like succession of day and night. Some of them have moons, that serve to give them light in the absence of the sun, as our moon does to us. They are all, in their motions, subject to the same law of gravitation as the earth is. From all this similitude, it is not unreasonable to think, that those planets may, like our earth, be the habitation of various orders of living creatures. There is some probability in this conclusion from analogy (Reid and Hamilton 1850, pp. 16-17)

In light of this quotation, let us pose a simple question: are the inferences one can make about black hole Hawking radiation by drawing on observations of dumb holes of the same kind as the inferences one can make about the existence of life on other planets by drawing on observations of life on Earth? We think not, and to make this clear we would like to draw a distinction between a notion of analogical reasoning, on the one hand, and a second notion of analogue simulation, on the other.

The literature on analogical reasoning is fairly extensive, with particularly noteworthy contributions by Keynes (Keynes 1921), Hesse (Hesse 1964, Hesse 1966) and Bartha (Bartha 2010, Bartha 2013). Drawing on this literature (in particular (Bartha 2013)), we can characterize analogical inferences in the following way: First, call the less accessible system about which we hope to make inferences the target, $T$, and call the more accessible system we hope to make use of in analogy the source, $S$. An argument using analogical reasoning then takes the following form (Bartha 2013):

P1. $S$ is similar to $T$ in certain known respects.

P2. $S$ has some further feature $Q$.

C. Therefore, $T$ also has the feature $Q$, or some feature $Q^{\star}$ that is similar to $Q$.

This seems to fit the Reid case rather well.

Following Hempel (Hempel 1965), the literature also recognizes a particular kind of argument by analogy, what Hempel called nomic isomorphism. Hempel characterized this kind of reasoning as a situation in which $S$ and $T$ are each governed by a set of laws, between which there is a syntactic isomorphism. When talking about syntactic isomorphism ' $[t]$ he essential idea is that the two sets of physical laws have a common 
mathematical form and may be obtained by assigning different physical interpretations to the symbols that appear in that common form' (Bartha 2010, pp. 208-9). ${ }^{5}$

These ideas offer a solid starting point for the philosophical analysis of the dumb hole case; however, several amendments and clarifications are required.

One central recommendation is that Hempel's notion of nomic isomorphism, which is a sub-species of analogical reasoning, should be replaced with a broader concept of analogue simulation. Analogue simulation, as we understand it, can occur even when the syntactic isomorphism one can identify does not hold between the laws governing the two systems in generality. This can be seen clearly in the dumb hole case since, strictly speaking, the equations on either side of the isomorphism are not laws. To establish a full nomic connection we would have to relate the laws governing the fundamental dynamics of quantum phenomena at the horizon of a black hole (i.e. the relevant equation from a prospective theory of quantum gravity) to those governing the fluid flow (i.e. at the very least the full Navier-Stokes equations). A doubly infeasible task.

Nevertheless, there is a syntactic isomorphism to be exploited in the dumb holes case, and we think it is best understood as holding between two very particular modelling frameworks, each with narrower scope than genuine laws. The question is not of an isomorphism between the laws of fluids and the laws of quantum gravity on the other. Rather, there is an isomorphism between a particular adequate way of modelling a special class of fluid setups and a particular adequate way of modelling the behaviour of quantum fields near a black hole horizon.

With this in mind let us introduce some vocabulary concerning models. We build models using a modelling framework, $M$. The ideal pendulum is a modelling framework for modelling particular pendulums. The two-body, point-particle modelling framework is good for modelling planetary orbits under certain conditions. Modelling frameworks almost always involve idealizations, and hence they are usually adequate only under a certain domain of conditions, $D$. The domain of conditions under which the ideal pendulum framework is adequate will depend on what we mean by adequate. In general, adequate means 'for a particular purpose: accurate to a certain desired degree.' People who use and build models have lots of background knowledge, some of it explicit and some of it tacit, about what domain of conditions needs to apply before a particular modelling framework is adequate for a particular purpose and to a particular desired degree of accuracy.

Given the above, we will say that system $S$ provides an analogue simulation of system $\mathrm{T}$ when the following set of conditions obtain: ${ }^{6}$

Step 1. For certain purposes and to a certain degree of desired accuracy, modelling framework $M_{S}$ is adequate for modelling system $S$ within a certain domain of conditions $D_{S}$.

\footnotetext{
${ }^{5}$ We retain Hempel's 'syntactic isomorphism' principally for historical reasons even though it is perhaps not the ideal terminology for the relationship at hand. The implication is always that the equations specifying two rather different models (say, the model of a simple pendulum with small displacement and the model of an RLC circuit) are 'the same'. This does not mean the equations are identical however. In an RLC circuit, for example, the equation for the resonance frequency is $\omega=(L C)^{-\frac{1}{2}}$, where $L$ is the inductance, and $C$ is the capacitance. In a simple pendulum, $\omega=(L g)^{-\frac{1}{2}}$ where $L$ is the length and $g$ is the acceleration of gravity. Those are not literally the same equation since one relates frequency to inductance and capacitance, etc. But we can easily see that they have the same structure - they are 'isomorphic' in syntax.

${ }^{6}$ The following is consistent with the account of simulation offered in (Winsberg 2009, Winsberg 2010)
} 
Step 2. For certain purposes and to a certain degree of desired accuracy, modelling framework $M_{T}$ is adequate for modelling system $T$ within a certain domain of conditions $D_{T}$.

Step 3. There exists exploitable mathematical similarities between the structure of $M_{S}$ and $M_{T}$ sufficient to define a syntactic isomorphism robust within the domains $D_{S}$ and $D_{T}$.

Step 4. We are interested in knowing something about the behaviour of a system $T$ within the domain of conditions $D_{T}$, and to a degree of accuracy and for a purpose consistent with those specified in Step 2. For whatever reasons, however, we are unable to directly observe the behaviour of a system $T$ in those conditions to the degree of accuracy we require.

Step 5. We are, on the other hand, able to study a system $S$ after having put it under such conditions as will enable us to conclude a statement of the form:

Claim $_{S}$ Under conditions $D_{S}$ and to degree of accuracy that will be needed below, we can for the purpose of employing the reasoning below assert that a system $S$ will exhibit phenomena $P_{S}$.

The formal similarities mentioned in Step 3 then allow us to reason from Claims to Claim $_{T}$ which is of the form:

Claim $_{T}$ Under conditions $D_{T}$ a system $T$ will exhibit phenomena $P_{T}$.

As a tool for analysing the structure of contemporary science this notion of simulation has the following advantages over the Hempelian category of nomic isomorphism. First, as we have already noted, the requirement that the syntactic isomorphism be between two sets of laws is too strong to cover most of the interesting cases. It seems clear that the kind of reasoning involved in dumb hole cases is more in the spirit of what Hempel had in mind than it is in the spirit of the sort of thing described by Keynes and Hesse, or of that exemplified by the Reid example. However, the dumb hole cases are difficult to see as fitting in to the strict set of requirements set out by Hempel. The relevant fact is not whether there is a formal relationship between two sets of laws, but rather whether such a relationship obtains between two suitably useful modelling frameworks.

Second, we think analogue simulation is better seen as distinct from analogical reasoning than as a sub-species of analogical reasoning. This is because the strength or quality of the inferences one can draw by analogue simulation is much greater than is that of those which can be drawn via analogical reasoning. We think analogue simulations can provide much stronger support for the conclusions we draw from them - this is of course the basis behind the key confirmation claims discussed at length in the following sections. Furthermore, we hope that our characterization of analogue simulation emphasizes the extent to which what we are dealing with is not simply a form of abstract argument, but rather a technique for learning about the world by manipulating it. It should be obvious, for example, that employing dumb holes to learn about Hawking radiation has a lot more in common with experiment than does Reid's armchair speculations about life on other planets. Step 5, above, after 
all, will require us to manipulate a system $S$ so as to put it in the set of conditions that allow us to make the inference to $\left[\mathrm{Claim}_{T}\right]$.

Third, we find it attractive that it is an easy consequence of our characterization of analogue simulation that analogue simulation and computer simulation come out as two species of the same genus: simulation. ${ }^{7}$ On our view, the main difference between computer simulation and analogue simulation is simply that in computer simulation, the system $S$ is a programmable digital computer, and the reasons that it meets the conditions articulated in Step 2 is that it has been programmed precisely so as to meet those conditions. The programmable digital computer is such a powerful scientific tool precisely because it can be so easily prepared in such a way. We find this consequence attractive, in part, because we find that it accords nicely with scientific practice and the intuitions of working scientists, who view the kind of work exemplified by dumb hole studies as easily comparable to computer simulations. We will comment further on the comparison with computer simulation in $\S 3.3$, once the major argument of the paper concerning confirmation has been introduced.

\subsection{Confirmation via Analogue Simulation}

The groundwork has now been laid for us to make our most controversial claim: that, in certain circumstances, analogue simulation can provide inductive support for a hypothesis regarding the target system, on the basis of empirical evidence regarding the source system - in other words, it can give us confirmation. It should be noted, that for the purpose of the present analysis we will not be concerned with the possibility of characterising cases of analogue simulation in terms of a particular philosophical model of confirmation. ${ }^{8}$ Rather, we will propose that certain cases of analogue simulation should plausibly be counted amongst the explananda for which the models of confirmation are intended to provide the explanans. From our perspective, if it proves that a philosophical model of confirmation cannot accommodate confirmation via analogue simulation at all, then this would be as much a problem for the model, as it would for analogue simulation.

That said, our aim here is emphatically not to propose a new category of confirmation based on analogue simulation merely on the basis of the intuitions and practices of 'working scientists'. Thus, we seek to carve a middle course between the normative and descriptive: neither assuming an abstract model of what should be counted by scientists as confirmatory, nor transcribing from their practice, a model of what actually is. We claim that philosophy of science is hostage to scientific practice and thus as the latter evolves, so must the former. That is, insofar as scientists develop novel methods for confirming hypotheses, our own models of confirmation must adapt to them. However, on our view, such novel practices must still be subjected to detailed philosophical analysis, and the cogency of claims of confirmation via novel methods tested all the more robustly on the grounds of their novelty. This is best done without prejudicing the analysis by adopting a particular account of confirmation. Only then,

\footnotetext{
${ }^{7}$ See (Winsberg 2010) for more on this point.

${ }^{8}$ Major approaches to confirmation theory (according to a relatively standard classification) are: confirmation by instances, hypothetico-deductivism, and probabilistic (Bayesian) approaches. See (Crupi 2013) for more details in general, and (Dizadji-Bahmani, Frigg, and Hartmann 2011) for work on applying the Bayesian framework in the context of analogical relationships. A forthcoming paper (Dardashti, Hartmann, Thébault, and Winsberg ) will explore the foundations of analogue simulation from a Bayesian persecutive.
} 
if the claim that a novel scientific practice is confirmatory survives such an analysis, can it be claimed that a philosophical model of confirmation needs to accommodate the new confirmatory mechanism.

Things of course also cut the other way. Nothing in the proposed methodology rules out the possibility that the scientist's claims (regarding the novel methods and confirmation) might either partially or entirely fail to live up to careful philosophical scrutiny. Our analysis, in such a case, would then licence normative arguments against the scientific intuitions. What is more, it could also be used to criticise philosophical models of confirmation for being overly permissive, rather than excluding new phenomena. In what follows we will, in fact, detail the extent to which 'confirmation via analogue simulation' can fail to hold. Thus, our analysis will also serve as a basis to identify cases which should not count amongst the explananda of models of confirmation, and we believe it is entirely possible that the existing philosophical models might be troubled by accommodating analogue simulation too easily.

The natural starting point for the discussion of confirmation in our specific case is an argument given by Bartha (Bartha 2013) against analogical reasoning being confirmatory. This argument is based upon a specific philosophical model of confirmation - Bayesian confirmation theory. However, in line with the considerations above, such a model will not be assumed in our positive story. From a Bayesian perspective it seems reasonable to assume that evidence for a hypothesis can count as confirmatory only if the probability of the hypothesis given the evidence together with certain background assumptions is larger than the probability of the hypothesis given only the background assumptions. Bartha contends that we should take the information encapsulated in an analogical argument to already be part of the background knowledge, and thus the probability of a hypothesis regarding the target system will be identical before and after finding any empirical evidence regarding the source system. It seems reasonable to accept this argument for the case in which the target and source are merely connected by analogical reasoning. However, when an analogical connection is established via analogue simulation there are good reasons to doubt the Bartha argument: prima facie, we do have the collection of new evidence - i.e. evidence which is not part of that background knowledge. Within our characterisation, this new evidence feature is found precisely when we probe the phenomenology of the source system to gain new evidence regarding an exemplar of the target system (i.e. Step 5). Of course, that this new evidence really is evidence relevant to the target system is only the case given the all important assumptions regarding the accuracy of the modelling frameworks and the robustness of the syntactic isomorphism (i.e. Steps 1-3). And clearly such assumptions are open to question without further support. Moreover, how can we be justified in thinking that the syntactic isomorphism will still hold within domains where we do not, by assumption, have any evidence that the modelling framework relevant to the target system is accurate?

Let us be more explicit regarding the required structure. The claim is that new evidence for the phenomena $P_{S}$ as predicted by the model $M_{S}$ of the system $S$, can confirm the existence of the analogous phenomena $P_{T}$, as predicted by the model $M_{T}$ of target system $T$. For such a claim to be justified not only must equivalence be established between the mathematical descriptions of $P_{S}$ and $P_{T}$, but we must also have evidence that both modelling frameworks will be accurate within the domain in which the phenomena are found. The problem of course is that it is not obvious how the accuracy of the framework $M_{T}$ within the domain $D_{T}$ could be established empirically with regard to the phenomena $P_{T}$, since we have assumed this phenomena to be 
inaccessible! Rather we need reasons external to the particular modelling frameworks at hand to justify the robustness of the formal correspondence.

We can see how this can be done as follows. Given certain explicit assumptions $\mathcal{A}=A_{1}, \ldots, A_{n}$ about the model $M_{T}$ of target system $T$, we are able to derive $P_{T}$. These assumptions are based on some additional implicit assumptions $\mathcal{I}=I_{1}, \ldots, I_{m}$ of the sort 'property $X$ of system $T$ does not influence the derivation'. These implicit assumptions are not "premises" of the derivation but are, if true, the justifications for the use of the assumptions $\mathcal{A}$. Now system $S$ is modelled in such a way as to realize the assumptions $\mathcal{A}^{\prime}=A_{1}^{\prime}, \ldots, A_{n}^{\prime}$, in model $M_{S}$. These assumptions are syntactically isomorphic to the assumptions $\mathcal{A}$ in model $M_{T}$. Therefore the derivation of $P_{S}$ goes through within model $M_{S}$ as it did in $M_{T}$ and we have a mathematical descriptions of the relevant phenomena which are suitably isomorphic. The set of underlying implicit assumptions $\mathcal{I}^{\prime}=I_{1}^{\prime}, \ldots, I_{k}^{\prime}$ for model $M_{S}$ can possibly be different from those of model $M_{T}$ and no syntactic isomorphism between the set of assumptions are needed here since they are not used in the derivation.

With respect to system $S$ one has control over the realization of the assumptions $\mathcal{A}^{\prime}$ necessary for the derivation, i.e. the required properties can to some extent be realized by construct. However, if system $T$ is inaccessible it remains an open question whether the assumptions are actually realized there. The reason for this is that the implicit assumptions $\mathcal{I}^{\prime}$ justifying the assumptions $\mathcal{A}^{\prime}$ in model $M_{S}$ are different from those justifying the assumptions $\mathcal{A}$ in model $M_{T}$. And knowledge about the $\mathcal{I}^{\prime}$ s do not necessarily entail any information about the implicit assumptions in the other system, since a priori these are independent. That is, in the worst case, one models in system $S$ something which is not realized in target system $T$.

So unless we have some reason to relate the implicit assumptions in both systems with each other there is no reason why observation of the phenomenon in one system should entail empirical evidence for the other. However, it is our claim, that in the case of analogue models of black hole Hawking radiation a relation between these implicit assumptions can be formulated which can be empirically tested and so build an empirical bridge between the target and source system.

Let us illustrate this with a simple example before discussing the dumbholes more closely in the next section. There is a syntactic isomorphism between Newton's Law of Gravity $F_{N}=G \frac{m_{1} m_{2}}{r^{2}}$ and Coulomb's law $F_{C}=K \frac{q_{1} q_{2}}{r^{2}} .{ }^{9}$ Let us, for the purpose of the argument, assume that Newton's law describes our inaccessible target system while we are able to test Coulomb's law. First note, how analogue simulation can obtain between systems with radically different ontologies. In one case we consider the strength of the interaction between charged objects while in the other case it is the strength of the interaction between massive bodies. But why should the force law between massive and charged objects be syntactically isomorphic?

One crucial formal feature behind the syntactic isomorphism is the dependence of the force law on the distance $r$ of the two bodies. In both cases we have a $1 / r^{2}$ dependence. One assumption that goes into the derivation of both laws and which gives rise to this dependence is the dimensionality of space. One can show that if space were to be 2-dimensional the force law in both cases would go as $1 / r$ while in four space dimensions the laws would go as $1 / r^{3}$. It is only in three space dimensions

\footnotetext{
${ }^{9}$ This example serves the purpose of exemplifying some of the aspects of analogue simulation but can actually not be applied to our framework since Coulomb's law describes static interactions only and so could not be used to test e.g. planetary motion.
} 
that the $1 / r^{2}$-relation obtains. ${ }^{10}$ This illustrates nicely how specific features, the dimensionality of space, common to both systems can lead to a syntactic isomorphism despite them having radically different ontologies.

The existence of such common features also serves as a guide to the conditions for the accuracy of the modelling frameworks and robustness of the isomorphism within the relevant domains. It is, in fact, clear from modern particle physics that not all fundamental interactions follow a $1 / r^{2}$-dependence in three spatial dimensions. ${ }^{11}$ In particular the weak nuclear force does not. Formally such features can be understood in the framework of quantum field theory (QFT) as relating to the mass of the force mediating bosons: QFT interactions are always mediated via so-called gauge bosons and a $1 / r^{2}$-dependence obtains only if we have massless-mediating particles. The gauge bosons of gravity and electromagnetism (the graviton and photon respectively) are massless, thus we get a $1 / r^{2}$-dependence. On the other hand the mediating bosons for the weak forces ( $W$ and $Z$ bosons) are massive, so the r-dependence is more complex. $^{12}$ The description of interactions via QFT is empirically well confirmed, and thus this argument towards the level of generality of the $1 / r^{2}$-dependence is not merely theoretical. It gives empirically grounded and model-external arguments for the robustness of the syntactic isomorphism between the Newtonian and Coulomb modelling frameworks ${ }^{13}$. Such additional knowledge coming from an underlying theory does not rule out the possibility of a breakdown in the syntactic isomorphism entirely, but it does give reason to insist the assumptions crucial to Steps 1-3 above are well-founded.

We have thus seen that additional knowledge of the underlying physics can give us reason to believe in the correctness of the underlying implicit assumptions. In the example considered we had shared explicit assumptions of the sort that both systems follow the Poisson equation in three space dimensions (for example) and implicit assumptions that the very specific properties of each of these systems do not lead to a deviation from the law. More precisely, we implicitly assume that the way electric charges interact is similar enough to the interaction of massive objects so that the $1 / r^{2}$ dependence in each case is robust. As we saw this assumption can be supported by model-external and empirically grounded arguments - let us abbreviate such arguments as 'MEEGA'. As we discussed above, the systems $T$ and $S$ differ in terms of the implicit assumptions. The non-realization of one of the implicit assumptions in the target system can lead to a failing of the analogue setup being able to confirm. MEEGA give us a handle on exactly these implicit assumptions and allow us to bridge in an empirically justifiable way the reasonableness of the implicit assumptions in the target system. Of course, this does not rule out the possibility that the violation of some unthought-of implicit assumption in the target system could lead to the target system not develop-

\footnotetext{
${ }^{10}$ Assuming the Poisson equation holds in all dimensions. There are also some other subtleties. See (Callender 2005) for details.

${ }^{11}$ There are physical proposals, like the Arkani-Hamed-Dvali-Dimopolous model (Arkani-Hamed, Dimopoulos, and Dvali 1998) or the Randall-Sundrum model (Randall and Sundrum 1999), which claim that there are additional dimensions. In the Randall-Sundrum model the claim that the dimensionality of space is a common feature of both gravitational and electromagnetic systems is denied: while electromagnetic interactions are restricted to three space dimensions, gravitational interactions are not. This shows that there is empirical room for these kind of modifications.

${ }^{12}$ For a simple treatment of this see Sec.1.4 and 1.5 in (Zee 2010).

${ }^{13}$ One might object that quantum field theory applies, unlike Newton's law of gravity, to fields and not particles. But this misses the point, since the relevant question is whether the same phenomena, namely the $1 / r^{2}$ relation in gravitational and electrostatic interactions are obtained. And since this is the case, QFT provides an independent justification for the robustness of the syntactic isomorphism.
} 
ing the phenomenon observed in the analogue system. Thus, MEEGA only ever give us an inductive base for believing in the robustness of the syntactic isomorphism and accuracy of the modelling frameworks, they do not establish anything tout court.

One might object that the establishment of MEEGA makes the need for the analogue simulation obsolete. This is not the case, since what MEEGA does is not to replace the modelling frameworks with an overarching theory but to justify empirically the validity of the implicit assumptions in each of the systems. As discussed above what a quantum field theoretic treatment is offering us is not a unifying theory of electromagnetic and gravitational interactions but constraints on any quantum field theoretical treatment of these interactions, thereby increasing our degree of believe with respect to the implicit assumptions and establishing a robustness of the modelling frameworks used. However, if there were one scientific theory that would cover both domains of applicability of the modelling frameworks, e.g. a unified theory of all fundamental forces, then, of course, the analogue simulation as a mean to confirmation becomes obsolete and we get back to standard theory confirmation by evidence.

So the lesson is, one is only justified in claiming confirmation via analogue simulation once one has established, via MEEGA, additional reasons for the accuracy of the modelling frameworks, and robustness of the syntactic isomorphism within the relevant domains. The key question examined in the remains of this paper is whether such conditions can be established for the case of dumb holes and black holes. However, before we enter into this discussion it will be instructive to review the crucial terminology and distinctions that have been introduced so far.

\subsection{Recapitulation}

There are three separate distinctions regarding types of scientific inference relevant to our argument. The first distinction is between ordinary analogical reasoning and analogue simulation and has already been discussed extensively in $\S 3.1$. The second distinction is between analogue simulation and computer simulation. Although analogical simulation is very much like computer simulation we do not here take them to be identical. There is a clear and obvious difference between the two in that in the case of computer simulation the structural similarity that exists between the model of the target and the model of the source exists precisely because the source system has been digitally programmed in such a way as to make this isomorphism obtain. However, there is a strong correspondence between such digital programming, and the preparation of the analogue model: the preparation of a fluid to formally resemble a black hole is, in a sense, a form of analogue programming (where here we are using 'analogue' in the 'not digital' sense of the word). We concede, therefore, that our claim that analogue simulation can confirm beliefs about the target simulation is not unrelated to the parallel claim about computer simulation, and we note that the latter claim is controversial in the literature. See (Beisbart and Norton 2012) for a defence of the negative claim, and (Parker 2009, Winsberg 2009), for a defence of the positive claim.

As it so happens, we take the positive side of this debate: since programmed digital computers are physical systems, a run of such a system gathers novel empirical evidence, and so surely must in principle be able to provide confirmation. Thus, on our view, and contra (Beisbart and Norton 2012), computer simulation is not simply an 'argument', and can in principle boost our degree of belief in a hypothesis about the target system, provided that the relevant background knowledge (concerning the 
isomorphism) is in place to support the relevant inference. Of course the run of a computer simulation will never serve to confirm all of the background knowledge supporting the claim of an existing structural similarity between source and target. A computer simulation of a fluid that uses the Navier-Stokes equations to guide its construction will never, by itself, confirm the Navier-Stokes equations themselves. But that does not mean that such a computer simulation cannot confirm, e.g., a scaling law regarding certain kinds of fluid configurations. ${ }^{14}$

This argument of course depends crucially upon the inference from the premise that programmed digital computers are physical systems, to the conclusion that a run of such a system can gather novel empirical evidence. Since, in such cases, the novel empirical evidence comes from a physical system whose job it is to perform calculations that could, in principle, have been carried out by rote on a piece of paper, a critic might - in support of (Beisbart and Norton 2012) - argue that we do not have genuinely novel empirical evidence in the sense that matters to confirmation theory. We believe that even if one accepts critical arguments in this vein, there are still cases of computer simulation that should plausibly be counted as confirmatory. And the reason why is, in fact, closely related to the idea of analogue simulation supported by model-external and empirically grounded arguments (i.e., MEEGA).

In practice much interesting science involves not just computer simulation in isolation, but the gathering of evidence regarding the reliability of the syntactic isomorphisms that such simulations exploit. Well-designed computer simulation studies generally involve a back-and-forth between simulation runs and real-world data gathering in support of their own background assumptions. Thus, certain cases of computer simulations involve the activity of providing MEEGA for the background assumptions used in other computer simulation, including, in some cases, themselves. In such cases, the role of simulation explicitly could not, even in principle, be played by calculations on a piece of paper, and thus the criticism of the evidence as not genuinely novel, falls short.

Thus, we do, as a matter of fact, think it is very plausible to believe that computer simulations can confirm certain hypotheses. However, this is not a central claim of this paper. One can reject the arguments in favour of computer simulations being confirmatory, but still accept that dumb hole experiments can confirm the existence of Hawking radiation. This is because of the third, and most important, distinction: the distinction between generic analogue simulations, on the one hand, and analogue simulation supported by MEEGA, on the other.

One might suspect that the arguments against computer simulation being confirmatory might be applicable to a general case of analogue simulation. However, as we have seen, such arguments are much less plausibly applicable in the case that computer simulations are engaged in, or supported by MEEGA. Similarly, for the case analogue simulation supported by MEEGA, the critical argument against the collection of novel evidence based upon the comparison with pen and paper calculation surely must fail entirely. In the case of an analogue simulation supported by MEEGA, by definition, there are model external, empirically grounded reasons to believe that novel

\footnotetext{
${ }^{14}$ To give a concrete example, it is widely held by astrophysicists that certain computer simulations carried out in the early 1970s (see for example (Toomre and Toomre 1972)), confirmed the previously heretical claim that tidal forces that arise when two galaxies collide were responsible for the phenomenon of galactic tails and bridges. We see no reason to deny this, since novel evidence came from the runs of of these simulations that rationally raised people's degrees of belief in the hypothesis. But of course, no one, on the other hand, would claim that these simulations could confirm the existence of tidal forces.
} 
phenomenology is being simulated. Thus, the collection of novel evidence is secured in a sense much stronger than that of either generic computer simulation or analogue simulation.

A further interesting point relates to the notion, mentioned above, of simulations which provide their own MEEGA. Such an idea will, in fact, prove to be embodied in precisely the case of analogue simulation under consideration. The central concern in this paper is the chain of arguments by which one might reasonably claim that dumb hole studies could confirm the existence of (gravitational) Hawking radiation. On our view, confirmation of Hawking radiation via analogue simulation can only be established given the acceptance of a chain of reasoning involving universality arguments in combination with diverse realisations of the counterpart effect. These diverse realizations will thus simultaneously provide the empirical support for the MEEGA supporting the simulation, and realize the simulations themselves. We will return to this point in $\S 4.2$.

\section{The Sound of Silence: Analogical Insights into Gravity}

\subsection{Experimental Realisation of Analogue Models}

The wish to use analogue models to test inaccessible target systems did not grew out of sheer creativity of the scientists involved but out of necessity. Science has reached a point where many theoretical ideas can not be tested due to several practical limitations. These limitations can have several reasons. For instance, the technology to test the theory has not yet been developed, the system that needs to be tested is unreachable, there is simply not enough funding to build the experiment, or a combination of the above. However, practical limitations to test a theory do not make a theory less scientific, and therefore the question remains of how then to establish confidence in these theories. In this context, analogue simulations have proved as a promising alternative and several applications beyond the dumb hole case, whose experimental realisation we will discuss now, have been proposed. Some further applications will be discussed in Sect. 5 .

If the analogue models of black hole physics were purely hypothetical then their scientific status would likely be merely that of a fascinating novelty, rather than the inspiration for an entire sub-field of modern physics. After all, the main problem with theoretical work concerning black holes is that we have, as yet, no empirical means of testing the predictions - since we can neither create black holes in the lab, nor probe them via astrophysical observation. In the spirit of our view of the dumb hole model as a simulation of a black hole, the situation, if one were not able to experimentally realise the model, would be like having the correct code for a computer simulation, but not being able to run it due to unrealistic hardware requirements. A not particularly useful situation. Here we will briefly survey the practical problem of detecting Hawking radiation via analogue simulation, and in doing so consider further models beyond the fluid mechanical dumb hole discussed above.

First let us consider a model along the lines of $\S 2.3$ with the flowing through a nozzle in order to create the deserved acoustic horizon. Following, (Novello, Visser, and Volovik 2002, p.26) we have that for supersonic flow of a fluid through a nozzle 
of radius $R$, the approximate value of $T_{H}^{\text {acoustic }}$ is given by the expression:

$$
T_{H}^{\text {acoustic }}=1.2 \times 10^{-6} \mathrm{~K}\left[\frac{c_{\text {sound }}}{1 \mathrm{~km} / \mathrm{s}}\right]\left[\frac{1 \mathrm{~mm}}{R}\right]
$$

For water this equates to a temperature of the order $10^{-6} \mathrm{~K}$. Detecting a thermal phonon spectrum at this temperature while the ambient temperature is approximately $300 \mathrm{~K}$ is entirely impractical, and so clearly water is not going to provide a useful working fluid for real laboratory experiments of this form. However, there are other methodologies for setting up the analogue model in which the use of conventional fluids for detecting at least classical aspects of Hawking radiation becomes more practicable (Weinfurtner, Tedford, Penrice, Unruh, and Lawrence 2011, Weinfurtner, Tedford, Penrice, Unruh, and Lawrenc 2013).

These experiments are based on a proposal by Schützhold and Unruh (Schützhold and Unruh 2002) where surface gravity waves in water tanks are used instead of Unruh's original proposal of sound waves. The problem for the sound wave proposal is that the acceleration of fluids to velocities close to the speed of sound leads to turbulences due to shock waves. Once there are turbulences the linearisation assumptions involved in the derivation of the effect are not realised anymore and experimental control of these turbulences is too difficult. The new proposal by Schützhold and Unruh considers surface waves on shallow water flows. The advantage is that the velocity of the background flow $v$ and the velocity of the surface waves $c$ are both dependent on the depth $h$ of the water tanks. More concretely, the velocity of the background flow goes as $v \propto \frac{1}{h}$, while the surface waves velocity goes as $c \propto \sqrt{h}$. So by adding an obstacle to the water flow, $h$ decreases, and thereby the background flow velocity increases while the surface wave velocity decreases. If the velocity of the background flow exceeds the velocity of the surface waves an effective horizon is obtained. ${ }^{15}$ The surface waves are effectively 'blocked' by the horizon, so nothing can enter the critical region. In this sense the experiment is a fluid mechanical analogue of a white hole, i.e. the time inverse of a black hole, for which the same laws hold.

The results of the experiment detailed in (Weinfurtner, Tedford, Penrice, Unruh, and Lawrenc 2013) imply that the pair-wave creation at the effective white hole horizon has a thermal spectra consistent with that predicted by the Hawking type calculation: the generated incoming positive mode is converted at the white hole horizon to positive and negative outgoing modes. And the corresponding amplitudes of the outgoing modes, i.e. the Bogoliubov coefficients, were measured and the validity of the thermal spectra according to Hawking checked. The dispersion relations used are different from the ones assumed by Hawking in his derivation which leads the authors of the paper to the following conclusion (p.15):

The ratio is thermal despite the different dispersion relation from that used by Hawking in his black hole derivation. This increases our trust in the ultraviolet independence of the effect, and our belief that the effect depends only on the low frequency, long wavelength aspects of the physics.

We will comment more on such issue regarding the relevance or not of short wavelength physics shortly. There are two drawbacks of this experiment. First, the experiment considers only the stimulated Hawking Process and not the spontaneous.

\footnotetext{
${ }^{15}$ The velocities needed to develop an effective horizon are low and the avoidance of turbulence is experimentally realisable and is being tested in the experiments.
} 
Second, only classical aspects of the process are measured in the experiment. The behaviour for this linear quantum system is dominated by the classical behaviour and additional quantum correlations of the emitted field excitations are not measured by the experiment. However, other analogue experiments based on Bose-Einstein condensates may be able to take the analogue experiments to the quantum regime.

The fluid mechanical analogy has proved to be only one among a number of possible realisations for the Hawking phenomena in terms of small oscillations in continuum systems. In general, it seems that there are only two necessary requirements to reproduce Hawking radiation (Barceló, Liberati, Visser, et al. 2005), in that we need: i) a quantum analogue model with a classical effective background and relativistic quantum fields living on it; which ii) contains some analogue geometry with some sort of horizon. Thus far, in addition to sound in a liquid and surface waves in water tanks, contemporary analogue gravity research (both theoretical and practical) makes use of: phonons in superfluid liquid helium, atomic Bose-Einstein condensates (BECs) (Garay, Anglin, Cirac, and Zoller 2000) and degenerate Fermi gases; 'slow light' in moving media; and traveling refractive index interfaces in nonlinear optical media (see (Carusotto, Fagnocchi, Recati, Balbinot, and Fabbri 2008) for further references).

\subsection{Universality and the Hawking Effect}

We must now turn the focus of our analysis to a problem of particular importance for both the reliability of the calculation of the Hawking effect, and our claim that the relevant analogue gravity models should be understood in terms of the concept of analogue simulation. In the standard calculation of the Hawking temperature, which is used in both the gravity and analogue cases, the black hole radiation (or its analogue) detected at late times (i.e. the outgoing particles) must be taken to correspond to extremely high frequency radiation at the horizon. This is because of an exponential gravitational red-shift (or its analogue) that is assumed to take place near the horizon. The problem with this is that the frequencies in question can in fact be high enough to make the relevant length scales smaller than those upon which the semi-classical approximation made in theories being applied are expected to work. For gravitation this corresponds to Planck-scale lengths at which it is no longer reasonable to use quantum fields defined upon fixed classical spacetime backgrounds. Such a 'trans-Planckian' regime is the dominion of theories of quantum gravity, and is thus well beyond the domain of applicability of the modelling framework we are using. This problem with trans-Planckian modes has a direct analogue in the fluids case, where the breakdown due to neglected quantum gravity effects is paralleled by that due to the atomic nature of the fluid. Thus, in each case the modelling framework we are using is in fact, strictly speaking, being applied beyond its proper domain of applicability. In and of itself, this is clearly a severe problem for the reliability of the Hawking radiation calculation in both the gravitational and the analogical situations. There is a sense in which such models fail the seemingly fundamental test of selfconsistency.

Furthermore, in light of the trans-Planckian problem (which we will use as a generic name for the breakdown of both gravitational and analogue models at small distances), it becomes questionable whether the notion of analogue simulation as we have defined it is really appropriate. Specifically, given these issues it seems reasonable to worry that both Steps 1 and 2 should be seen to fail, and thus that we have inappropriately applied our own concept of analogue simulation! Rather, perhaps, 
the appropriate philosophical framework for dumb hole models is something like analogical reasoning, as traditionally conceived. And for this case at least, our efforts in introducing the new conceptual framework of analogue simulation have been in vain.

Fortunately, things are not quite as bad as they seem. As we shall see, although not entirely solved, the trans-Planckian problem can be reformulated such that we can give precise conditions under which Hawking effect calculations are reliable. The relevant definitions of the modelling frameworks and domains of applicability can then be appropriately amended, and both Hawking radiation and analogue simulation can be saved from the trans-Planckian spectre. Before we consider such more sophisticated arguments regarding the relevance of trans-Planckian effects, it will prove particularly interesting, from an analogical reasoning perspective, to first consider the chain of theoretical developments that lead to the formal arguments that will be presented. Let us start with a key observation by Unruh regarding the differing epistemological statuses of the two breakdowns:

At wavelengths shorter than the inter-atomic spacing, sound waves do not exist and thus the naive derivation of the temperature of dumb holes will fail. But unlike for black holes, for dumb holes, the theory of physics at short wavelengths, the atomic theory of matter, is well established. For black holes, a quantum theory of gravity is still a dream. Thus, if one could show that for dumb holes the existence of the changes in the theory at short wavelengths did not destroy the existence of thermal radiation from a dumb hole, one would have far more faith that whatever changes in the theory quantum gravity created, whatever nonlinearities quantum gravity introduced into the theory, the prediction of the thermal radiation from black holes was robust. (Unruh 2008, p.2908)

This is of course a beautiful example of analogical reasoning, perhaps more attractive, but of the same genus to that of Reid. If we could show that the trans-Planckian fluid dynamical effects are irrelevant in our model of the fluid, we could speculate the same may be true with the gravitational case also. Even if we cannot make use of a precise mathematical relationship between the two situations to make an inferences à la analogue simulation, we could use the knowledge that they are similar in certain respects to make a less reliable inference à la analogical reasoning.

A specific suggestion regarding modelling of the breakdown for the fluid mechanical case was in fact made by Jacobson (Jacobson 1991, Jacobson 1993) in the early nineties: one can focus upon the altered dispersion relation (i.e. relationship between frequency and wavenumber) that is relevant to an atomic fluid rather than continuous fluid, and consider whether, in such models, the exponential relationship actually holds between the outgoing wave at some time after the formation of the horizon, and the wavenumber of the wave packet (Unruh 2008). Approximate answers to such questions can be determined in practice via numerical methods, and it was shown by Unruh in 1995 that the altered dispersion relation in atomic fluids does imply that the early time quantum fluctuations that cause the late-time radiation are not in fact exponentially large (Unruh 1995). Thus, there is sufficient basis to analogically reason that the trans-Planckian alterations to the gravitational dispersion relationship might also prove irrelevant to the Hawking effect. However, the situation has in fact proved much better than this: we can reasonably establish the applicability of full analogue simulation despite trans-Planckian effects in both systems. Recent work in fact allows 
us to generalise from the specific fluid dynamical alterations to the dispersion relation, to a model with a generically altered relation, independent of the particular cause of the trans-Planckian breakdown. Of particular relevance are calculations to this end by Unruh and Schützhold (Unruh and Schützhold 2005). Their results represent a generalisation of earlier work by Corley (Corley 1998) and provide a basis for a universality claim with regard to the Hawking effect. ${ }^{16}$ Unruh and Schützhold demonstrate that specific assumptions and approximations can be made such that the role of possible trans-Planckian effects is factored into the calculation of Hawking radiation, and that, for such cases, the additional effects are found not to disturb the thermal spectrum as originally derived by Hawking. The Hawking effect does not, to lowest order, depend on the details of the dispersion relation at high wave numbers given certain modelling assumptions.

What is more desirable, however, is a set of general conditions under which such effective decoupling between the sub- and trans- Planckian physics can be argued to take place. Unruh and Schützhold's proposal in this vein runs as follows: we assume the breakdown of geometric optics (leading to the creation of particles) occurs in the vicinity of the horizon only. There the gravitational redshift induces a transition of trans-Planckian into sub-Planckian modes. Given this assumption, if the modes leave the Planckian regime in their ground state with respect to freely falling observers ${ }^{17}$ near the horizon, then Hawking radiation can be obtained (Unruh and Schützhold 2005 , p.8). The condition of the modes leaving the Planckian regime in their ground state with respect to freely falling observers is then taken to obtain in circumstances which: a) there is a privileged freely falling frame (in line with the breaking of Lorentz invariance at the Planck scale); b) the Planckian modes start off in their ground state; and c) the evolution of the modes is adiabatic and therefore the Planckian dynamics is understood to be much faster then all external variations.

We thus see that there are good theoretical reasons for a qualified claim that Hawking radiation is a universal effect. Such claims provide a model-external basis for both the accuracy of the modelling frameworks and the robustness of the syntactic isomorphism within the domains of the gravitational and fluid mechanical Hawking phenomena. However, in order for such external lines of reasoning to count as genuine justification we earlier insisted that they be empirically grounded. How can we do this for our universality arguments? The answer is quite simple: we can empirically ground the universality argument vis-à-vis gravitational and fluid mechanical Hawking radiation, by empirically grounding the same argument as applied to other pairs of analogue models. Replication of Hawking phenomena could be achieved experimentally in systems as different as phonons in superfluid liquid helium, or traveling refractive index interfaces in nonlinear optical media. The fact that each system has a different underlying microphysics then gives empirical reasons to support the universality claim. This claim then justifies assuming the accuracy of the modelling frameworks and robustness of the syntactic isomorphism for the gravitational-acoustic case.

It is important to realize that the universality claim is not a theory replacing the modelling frameworks themselves. It is not a theory of slow light in dielectric media, sound in fluids and quantum fields around a black hole horizon at the same time.

\footnotetext{
${ }^{16}$ For further work on these issues, using a range of different methodologies, see for example (Himemoto and Tanaka 2000, Barceló, Garay, and Jannes 2009, Coutant, Parentani, and Finazzi 2012).

${ }^{17}$ For the dumb hole case the freely falling frame corresponds to the local rest frame of the fluid flow.
} 
The sole purpose of the universality claim is to establish the independence of the Hawking radiation derivation in all these different systems from possible high energy physics effects, whatever these may be. And so, once it is empirically grounded, it establishes the validity of the most crucial implicit assumption in the derivation of the phenomenon.

\subsection{Confirmation of Gravitational Hawking Radiation}

All the features of our argument towards the possibility of confirmation of gravitational Hawking radiation via analogue simulation are now assembled. We are now in the position to make the central, and we believe rather bold, claim of the paper: Current and future experimental evidence of Hawking radiation from dumb holes can provide a high degree of warrant to claims regarding the existence of the phenomena in gravitational systems. Confirmation in the one case, can be understood as constituting confirmation in the other. We can proceed towards the establishment of this claim as follows:

Step 1. For certain purposes and to a certain degree of desired accuracy, modelling framework $M_{S}$ is adequate for modelling systems of type $S$ within a certain domain of conditions $D_{S}$.

In our case, a system of type $S$ will be a fluid flow set up. The modelling framework $M_{S}$ will consist of treating a fluid as a continuous, compressible, inviscid medium, without relativistic effect, and under the conditions of being barotropic and locally irrotational, along with the three conditions mentioned in $\S 2.3-$ and then quantizing the linearized fluctuations. The domain of conditions $D_{S}$ under which we consider $M_{S}$ to be adequate for modelling systems of type $S$ for the purpose of calculating an acoustic Hawking temperature high enough to be detectable under reasonable laboratory conditions are the ones in which the fluid is set up with a flow so as to have an 'acoustic horizon' of the various kinds we talked about in Section 4.1. The work in dealing with the transPlankian problem discussed above is sufficient to demonstrate the viability of the modelling framework for some further refinement of $D_{S}$.

Step 2. For certain purposes and to a certain degree of desired accuracy, modelling framework $M_{T}$ is adequate for modelling systems of type $T$ within a certain domain of conditions $D_{T}$.

The system of type $T$ in this case is of course the astrophysical black hole. The modelling framework $M_{T}$ is a semi-classical model for gravity in which we have: i) a fixed classical spacetime that features the establishment of an event horizon via gravitational collapse leading to a black hole; and ii) a quantum scalar field evaluated in the regions of past and future null infinity which are assumed to be Minkowskian. The domain of conditions $D_{T}$ is limited to the times after the collapse phase of the black hole, the 
details of which are assumed to be irrelevant. The work in dealing with the trans-Plankian problem discussed above is sufficient to demonstrate the viability of the modelling framework for some further refinement of $D_{S}$. For example the conditions a-c proposed by Unruh and Schützhold and discussed above.

Step 3. There exists exploitable mathematical similarities between the structure of $M_{S}$ and $M_{T}$ sufficient to define a syntactic isomorphism robust within the domains $D_{S}$ and $D_{T}$.

This step essentially follows from the two steps above given the refinements of $D_{S}$ and $D_{T}$ regarding the trans-Plankian issue. Given both theoretical arguments towards universality of Hawking radiation (which do exist) and empirical support for these arguments (which currently does not), we have justification for assuming the accuracy of the modelling frameworks within the relevant domains, and, furthermore, robustness of the syntactic isomorphism.

Step 4. We are interested in knowing something about the behaviour of a system of type $T$ within the domain of conditions $D_{T}$, and to a degree of accuracy and for a purpose consistent with those specified in Step 2. For whatever reasons, however, we are unable to directly observe the behaviour of an exemplar of a system $T$ in those conditions to the degree of accuracy we require.

We want to know whether black holes exhibit Hawking radiation (presumably to a degree of accuracy that is sufficient for the purpose of warranting various speculations about black hole thermodynamics, etc.) but we can neither create black holes in the lab, nor probe them via astrophysical observation.

Step 5. We are, on the other hand, able to study an exemplar of a system of type $S$ after having put it under such conditions as will enable us to conclude a statement of the form:

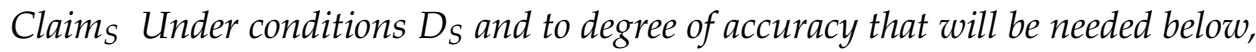
we can for the purpose of employing the reasoning below assert that a system of type $S$ will exhibit phenomena $P_{S}$.

We build an acoustic system of type $S$ that meets conditions $D_{S}$ and we successfully experimentally measure (confirming it to be genuine signal rather than noise) the acoustic Hawking Radiation predicted by $M_{S}$.

The formal similarities mentioned in Step 3 then allow us to reason from the existence of acoustic Hawking Radiation in dumb holes to Hawking radiation in black holes. From this we progress to our key claim: confirmation via observation under conditions $D_{S}$ of the existence of acoustic Hawking Radiation would allow us to speak of having confirmed the existence of the analogue phenomenon, gravitational Hawking Radiation. Mutatis mutandis for disconfirmation. Thus we see that so far as Hawking radiation goes, and given some external support via MEEGA, dumb holes have the potential to tell us rather a lot about gravity. 


\section{Prospectus}

The dumb hole/black hole case is a powerful illustration of the relevance of the notion of analogue simulation to modern science, and we believe there is a large range of further possible applications of the idea. A non-exhaustivity list of prospective cases is: 1) use of the AdS/CFT correspondence to make predictions regarding quarkgluon plasma, super conductors and super fluids (Hartnoll, Herzog, and Horowitz 2008, Faulkner, Iqbal, Liu, McGreevy, and Vegh 2010); 2) use of two-component BoseEinstein condensates to study cosmic inflation (Fischer and Schützhold 2004); 3) use of trapped-ions to simulate neutrino oscillation (Noh, Rodríguez-Lara, and Angelakis $2012)$; 4) there are many applications of analogue simulation in condensed matter systems due to difficulties in solving quantum many-body problems, e.g. simulating high-temperature superconductors through quantum-dot arrays (Manousakis 2002), quantum phase transitions from a superfluid to a Mott insulator phase through trapped atoms in an optical lattice (Greiner, Mandel, Esslinger, Hänsch, and Bloch 2002a) and many more ${ }^{18}$.

Amongst the field of such potential examples, what seems the most straight forward candidate for analogue simulation in contemporary science is the simulation of one quantum system by another. This general idea goes back to Feynman (Feynman 1982, Feynman 1986), and has rather diverse applications in terms of both quantum simulation via a programmable quantum computer ${ }^{19}$ and via a quantum analogue system..$^{20}$ Focussing on the latter, the essential idea is to manipulate a well controlled quantum system - such as atoms in an optical trap - such that the Hamiltonian evolution of a different system is implemented. One then aims to probe the properties of the relevant Hamiltonian, and in doing so produce experimental measurements of phenomena whose correlates in the target system are experimentally inaccessible.

A particularly impressive recent example is the simulation of 'Zitterbewegung' (trembling motion) phenomena long known to be predicted by the Dirac equation (Schrödinger 1930), using a single trapped ion set to behave as an analogue to a free relativistic quantum particle (Gerritsma, Kirchmair, Zähringer, Solano, Blatt, and Roos 2010). As is noted by the relevant authors, this case bears a strong resemblance to the dumb hole simulation of Hawking radiation. It is also a good illustration of the idea of analogue simulation that we have articulated: we have two theories, nonrelativistic quantum mechanics, according to the Schrödinger equation, and relativistic quantum mechanics, according to the Dirac equation. And we have an experimentally inaccessible novel phenomena, Zitterbewegung, which is predicted by one theory. Then, in certain very specific circumstances, we have an isomorphism between the two theories which allows us to experimentally simulate certain relevant formal structures, the Dirac equation in 1+1 dimensions, and thus reproduce a correlate of the novel phenomena. Given isolation of suitable MEEGA, confirmation via analogue simulation could then be understood to function as with the Hawking radiation case. More detailed investigation of this and other cases will be conducted in future work.

\footnotetext{
${ }^{18}$ See (Georgescu, Ashhab, and Nori 2013) for a recent review.

${ }^{19}$ See (Deutsch 1985, Bernstein and Vazirani 1993, Shor 1997) for key historical developments, and see (Nielsen and Chuang 2010, Timpson 2013) for general reference.

${ }^{20}$ See (Lloyd 1996, Cirac and Zoller 2012, Greiner, Mandel, Esslinger, Hänsch, and Bloch 2002b, Ma, Dakic, Naylor, Zeilinger, and Walther 2011) for more details of both the concept and its applications.
} 


\section{Funding}

E.W. is grateful for the hospitality of the Center for Advanced Studies and Munich Center for Mathematical Philosophy (both Ludwig Maximilian Universität Munich) in providing a visiting fellowship during the summer of 2013. R.D. and K.T. would like to acknowledge the support of the Alexander von Humboldt foundation.

\section{Acknowledgements}

We would like to thank Michael Berry, Jeremy Butterfield, Erik Curiel, Richard Dawid, Stephan Hartmann, Sebastian Lutz and William Unruh for various comments, discussions and suggestions that greatly helped in the development of this paper. We are also appreciative of the input of three anonymous referees, and of audiences at the workshop 'Reduction and Emergence in Physics' (Munich 2013), the 'British Society for the Philosophy of Science Annual Conference' (Cambridge 2014), and the 'University of Geneva Workshop on Models and Experiments' (Geneva 2014).

\section{References}

Arkani-Hamed, N., S. Dimopoulos, and G. Dvali (1998). 'The hierarchy problem and new dimensions at a millimeter'. Physics Letters B 429(3), pp. 263-72.

Barceló, C., L. J. Garay, and G. Jannes (2009). 'Sensitivity of Hawking radiation to superluminal dispersion relations'. Physical Review D 79(2), p. 024016.

Barceló, C., S. Liberati, M. Visser, et al. (2005). 'Analogue gravity'. Living Rev. Rel 8(12).

Bartha, P. (2013). 'analogy and analogical reasoning'. In E. N. Zalta (Ed.), The Stanford Encyclopedia of Philosophy (Fall 2013 ed.).

Bartha, P. F. (2010). By parallel reasoning: The construction and evaluation of analogical arguments. Oxford: Oxford University Press.

Beisbart, C. and J. D. Norton (2012). 'Why Monte Carlo Simulations Are Inferences and Not Experiments'. International Studies in the Philosophy of Science 26(4), pp. $403-22$.

Bernstein, E. and U. Vazirani (1993). 'Quantum complexity theory'. In Proceedings of the twenty-fifth annual ACM symposium on Theory of computing, pp. 11-20. ACM.

Callender, C. (2005). 'Answers in search of a question:'proofs' of the tridimensionality of space'. Studies in History and Philosophy of Science Part B: Studies in History and Philosophy of Modern Physics 36(1), pp. 113-36.

Carusotto, I., S. Fagnocchi, A. Recati, R. Balbinot, and A. Fabbri (2008). ‘Numerical observation of Hawking radiation from acoustic black holes in atomic BoseEinstein condensates'. New Journal of Physics 10(10), p. 103001.

Cirac, J. I. and P. Zoller (2012). 'goals and opportunities in quantum simulation'. Nature Physics 8(4), pp. 264-6.

Corley, S. (1998). 'Computing the spectrum of black hole radiation in the presence of high frequency dispersion: An analytical approach'. Physical Review D 57(10), p. 6280. 
Coutant, A., R. Parentani, and S. Finazzi (2012). 'Black hole radiation with short distance dispersion, an analytical S-matrix approach'. Physical Review D 85(2), p. 024021.

Crupi, V. (2013). Confirmation. In E. N. Zalta (Ed.), The Stanford Encyclopedia of Philosophy (Winter 2013 ed.).

Dardashti, R., S. Hartmann, K. Thébault, and E. Winsberg. Confirmation via Analogue Simulation: A Bayesian Account. (in progress).

Deutsch, D. (1985). 'Quantum theory, the Church-Turing principle and the universal quantum computer'. Proceedings of the Royal Society of London. A. Mathematical and Physical Sciences 400(1818), pp. 97-117.

Dizadji-Bahmani, F., R. Frigg, and S. Hartmann (2011). 'Confirmation and reduction: a Bayesian account'. Synthese 179(2), pp. 321-38.

Faulkner, T., N. Iqbal, H. Liu, J. McGreevy, and D. Vegh (2010). 'From black holes to strange metals'. arXiv preprint arXiv:1003.1728.

Feynman, R. P. (1982). 'Simulating physics with computers'. International journal of theoretical physics 21(6), pp. 467-88.

Feynman, R. P. (1986). 'Quantum mechanical computers'. Foundations of physics 16(6), pp. 507-31.

Fischer, U. R. and R. Schützhold (2004). 'Quantum simulation of cosmic inflation in two-component Bose-Einstein condensates'. Physical Review A 70(6), p. 063615.

Garay, L., J. Anglin, J. Cirac, and P. Zoller (2000). 'Sonic analog of gravitational black holes in Bose-Einstein condensates'. Physical Review Letters 85(22), p. 4643.

Georgescu, I., S. Ashhab, and F. Nori (2013). 'Quantum simulation'. arXiv preprint arXiv:1308.6253.

Gerritsma, R., G. Kirchmair, F. Zähringer, E. Solano, R. Blatt, and C. Roos (2010). 'Quantum simulation of the Dirac equation'. Nature 463(7277), 68-71.

Greiner, M., O. Mandel, T. Esslinger, T. W. Hänsch, and I. Bloch (2002a). 'Quantum phase transition from a superfluid to a Mott insulator in a gas of ultracold atoms'. Nature 415(6867), 39-44.

Greiner, M., O. Mandel, T. Esslinger, T. W. Hänsch, and I. Bloch (2002b). 'Quantum phase transition from a superfluid to a Mott insulator in a gas of ultracold atoms'. Nature 415(6867), 39-44.

Hartnoll, S. A., C. P. Herzog, and G. T. Horowitz (2008). 'Building an AdS/CFT superconductor'. arXiv preprint arXiv:0803.3295.

Hawking, S. W. (1975). 'Particle creation by black holes'. Communications in mathematical physics 43(3), 199-220.

Hempel, C. G. (1965). Aspects of Scientific Explanation and other Essays in the Philosophy of Science. New York: Free Press.

Hesse, M. (1964). 'Analogy and Confirmation Theory'. Philosophy of Science 31, pp. $319-27$.

Hesse, M. B. (1966). Models and Analogies in Science, Volume 7. Notre Dame: University of Notre Dame Press. 
Himemoto, Y. and T. Tanaka (2000). 'Generalization of the model of Hawking radiation with modified high frequency dispersion relation'. Physical Review D 61(6), p. 064004.

Jacobson, T. (1991). 'Black-hole evaporation and ultrashort distances'. Physical Review D 44(6), p. 1731.

Jacobson, T. (1993). 'Black hole radiation in the presence of a short distance cutoff'. Physical Review D 48(2), pp. 728-41.

Jacobson, T. (1996). 'introductory lectures on black hole thermodynamics'.

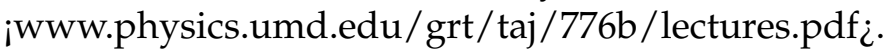

Keynes, J. M. (1921). A Treatise on Probability. London: Macmillan \& Co.

Landau, L. and E. Lifshitz (1987). Fluid Mechanics. Oxford: Butterworth-Heinemann.

Leonhardt, U. and T. G. Philbin (2008). 'The case for artificial black holes'. Philosophical Transactions of the Royal Society A: Mathematical, Physical and Engineering Sciences 366(1877), pp. 2851-7.

Lloyd, S. (1996). 'Universal Quantum Simulators'. Science 273, 1073-7.

Ma, X.-s., B. Dakic, W. Naylor, A. Zeilinger, and P. Walther (2011). 'Quantum simulation of the wavefunction to probe frustrated Heisenberg spin systems'. Nature Physics 7(5), 399-405.

Manousakis, E. (2002). 'A quantum-dot array as model for copper-oxide superconductors: A dedicated quantum simulator for the many-fermion problem'. Journal of low temperature physics 126(5-6), 1501-13.

Mukhanov, V. and S. Winitzki (2007). Introduction to quantum effects in gravity. Cambridge: Cambridge University Press.

Nielsen, M. A. and I. L. Chuang (2010). Quantum computation and quantum information. Cambridge: Cambridge University Press.

Noh, C., B. Rodríguez-Lara, and D. G. Angelakis (2012). 'Quantum simulation of neutrino oscillations with trapped ions'. New Journal of Physics 14(3), 033028.

Novello, M., M. Visser, and G. E. Volovik (2002). Artificial black holes. Singapore: World Scientific Singapore.

Parker, W. S. (2009). 'Does matter really matter? Computer simulations, experiments, and materiality'. Synthese 169(3), 483-96.

Randall, L. and R. Sundrum (1999). 'Large mass hierarchy from a small extra dimension'. Physical Review Letters 83(17), p. 3370.

Reid, T. and W. Hamilton (1850). Essays on the intellectual powers of man. Cambridge: J. Bartlett.

Schrödinger, E. (1930). Über die kräftefreie Bewegung in der relativistischen Quantenmechanik. Akademie der wissenschaften in kommission bei W. de Gruyter u. Company.

Schützhold, R. and W. G. Unruh (2002). 'Gravity wave analogues of black holes'. Physical Review D 66(4), p. 044019.

Shor, P. W. (1997). 'Polynomial-time algorithms for prime factorization and discrete logarithms on a quantum computer'. SIAM journal on computing 26(5), 1484-509. 
Thompson, R. and L. Ford (2008). 'Enhanced black hole horizon fluctuations'. Physical Review D 78(2), p. 024014.

Timpson, C. G. (2013). Quantum Information Theory \& the Foundations of Quantum Mechanics. Oxford: Oxford: Oxford University Press.

Toomre, A. and J. Toomre (1972, December). 'Galactic Bridges and Tails'. Astrophysical Journal 178, pp. 623-66.

Unruh, W. (1981). 'Experimental black-hole evaporation?'. Physical Review Letters 46(21), pp. 1351-53.

Unruh, W. (1995). 'Sonic analogue of black holes and the effects of high frequencies on black hole evaporation'. Physical Review D 51(6), p. 2827.

Unruh, W. (2008). 'Dumb holes: analogues for black holes'. Philosophical Transactions of the Royal Society A: Mathematical, Physical and Engineering Sciences 366(1877), pp. 2905-13.

Unruh, W. G. (2014). Has hawking radiation been measured? Foundations of Physics 44(5), 532-545.

Unruh, W. G. and R. Schützhold (2005). 'Universality of the Hawking effect'. Physical Review D 71(2), p. 024028.

Weinfurtner, S., E. W. Tedford, M. C. Penrice, W. G. Unruh, and G. A. Lawrenc (2013). 'Classical aspects of Hawking radiation verified in analogue gravity experiment'. arXiv preprint arXiv:1302.0375.

Weinfurtner, S., E. W. Tedford, M. C. Penrice, W. G. Unruh, and G. A. Lawrence (2011). 'Measurement of stimulated Hawking emission in an analogue system'. Physical Review Letters 106(2), p. 021302.

Winsberg, E. (2009). 'A tale of two methods'. Synthese 169(3), pp. 575-92.

Winsberg, E. (2010). Science in the age of computer simulation. Chicago: University of Chicago Press.

Zee, A. (2010). Quantum Field Theory in a Nutshell (2nd ed.). Princeton: Princeton University Press. 